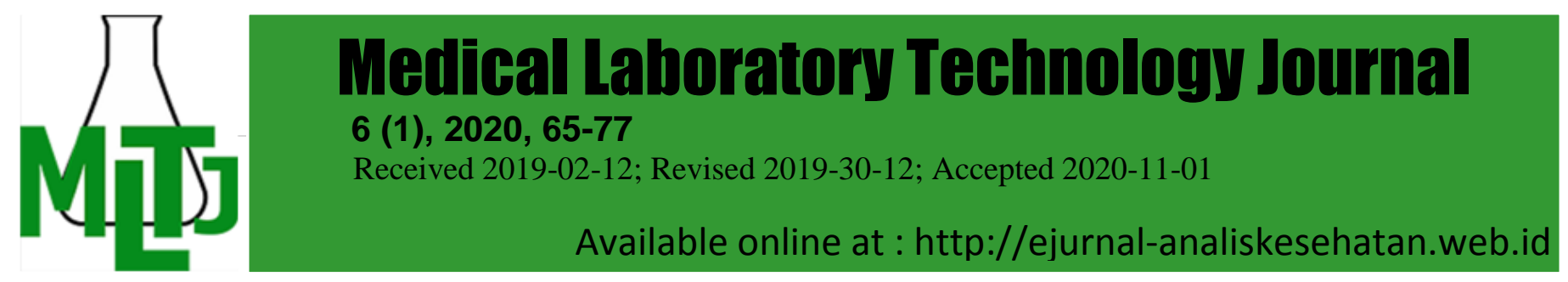

\title{
The Effect of Dehydroepiandrosterone on Ovarian Reserve in Ovarian Damage Caused by Methotrexate
}

\author{
Ali Doğukan Anğın ${ }^{1}$, Ebru Çöğendez ${ }^{2}$, İsmet Gün ${ }^{3}$, Önder Sakin ${ }^{1}$, Muzaffer \\ Seyhan Çıkman ${ }^{1}$, Kayhan Başak ${ }^{1}$, Asuman Orçun Kaptanağası ${ }^{1}$, Yasemin \\ Alan", Murat Alan5, *Emrah Beyan ${ }^{5}$
}

${ }^{1}$ Dr Lütfi Kırdar Kartal Training and Research Hospital, Department of Obstetrics and Gynecology, University of Health Sciences, Turkey. 'Zeynep Kamil Women's and Children's Disease Training and Research Hospital, Department of Obstetrics and Gynecology, University of Health Sciences, Turkey. ${ }^{3}$ Sultan Abdülhamid Han

Training and Research Hospital, Department of Obstetrics and Gynecology, University of Health Sciences, Turkey. ${ }^{4}$ Department of Obstetrics and Gynecology, İzmir Metropolitan Municipality Eşrefpaşa Hospital, Turkey. ${ }^{5}$ Tepecik Education and Research Hospital, Department of Obstetrics and Gynecology, University of Health Sciences, Turkey. *Email: emrahbeyan@gmail.com

DOI: $10.31964 / \mathrm{mltj} . v 1 \mathrm{i1} .270$

\begin{abstract}
To determine the possible detrimental effects of multiple methotrexate doses has on the ovarian reserve and to determine the beneficial effects of dehydroepiandrosterone supplementation. The rats $(\mathrm{n}: 24)$ divided into three groups; Group 1: control group, Group 2: dehydroepiandrosterone and methotrexate group $(6 \mathrm{mg} / \mathrm{kg}$ dehydroepiandrosterone dissolved in $0.1 \mathrm{ml}$ sesame seed oil subcutaneously for ten days and $1 \mathrm{mg} / \mathrm{kg}$ intramuscular methotrexate at the 1st, 3rd, 5th and 7th days) and Group 3: methotrexate group (1mg/kg intramuscular methotrexate at the 1st, 3rd, 5th and 7th day). The groups compared in regards to their histopathological ovarian damage scores and $\mathrm{AMH}$ values. It established that multiple methotrexate applications had a considerable effect on reducing vascular congestion in the ovarian tissue. Both in groups 2 and $3 \mathrm{AMH}$ values found to be significantly lower. When this decline in the ovarian reserve examined comparatively; while both the group 2 and 3 reported having a considerable and continuous reduction in the $\mathrm{AMH}$ levels correlative to the control group; the primordial, primary and total follicle counts shown to stay statistically the same in the group 2 $(p<0.0167)$. The dehydroepiandrosterone supplementation, while did not have a significant effect on reducing ovarian damage induced by methotrexate, also was not shown to provide any considerable increase in $\mathrm{AMH}$ levels. However, prolonged usage of dehydroepiandrosterone by contributing to the antral follicle pool considered promising in increasing the $\mathrm{AMH}$ levels conceivably.
\end{abstract}

Keywords: dehydroepiandrosterone, methotrexate, $\mathrm{AMH}$, ovary, reserve

\section{INTRODUCTION}

Adjuvant chemotherapy is a widely applied medical treatment of gynecological malignancies. Chemotherapeutic agents have a toxic effect on gonads on varying degrees, and in turn, this is having a detrimental impact on ovarian reserve. The risk of ovarian insufficiency directly correlated with the mechanism of action of the agent used. The cumulative dosage, the duration of the treatment, and the age of the patients during treatment. It should note that with the younger patients, ovarian 
reserves are in a considerably better state comparative to the patients over 40 years old. Lately, the effect of chemotherapy on the reproduction capacities and particularly the ovarian reserves have become even more debatable; because the number of patients who survives hematological malignancies and breast cancer has increased (Howell \& Shalet, 1998).

Another area where a chemotherapeutic agent such as Methotrexate is widely applied, besides the malignancies, is ectopic pregnancies (EP) (Lipscomb, 2007). EP is a significant condition that carries high morbidity and mortality rates, and depending upon the method of treatment may impact the future fertility potential of the patient. The decline in fertility due to loss of ovarian reserve. Resulting from; the condition itself (tubular rupture), the surgical treatment, the medical treatments utilizing Methotrexate, is a circumstance that needs further deliberation nowadays.

The irreversible loss of the oocytes due to chemotherapy has brought upon new approaches to better conserve the fertility in these patients. Currently, the options on preserving fertility are the cryopreservation of oocyte and ovarian tissue and the freezing of embryo (Wallace, 2011). On a young patient with a healthy ovarian reserve, cryopreservation of oocyte may impact the future fertility of the patient positively. However, the main problem is with cases that receive chemotherapy before the cryopreservation of oocyte or ovarian tissue and with the facts that have insufficient ovarian reserves before treatment. In 2000, Casson and colleagues were the first groups to suggest DHEA supplementation to increase ovarian response in women with diminished ovarian reserves (DOR)(Casson, 2000). As a precursor of estradiol (E2) and testosterone, DHEA originates from the zona reticularis layer of the adrenal cortex and the theca cells of the ovary (Burger, 2002; Luchetti et al., 2004; Millier, Whitelaw, \& Smyth, 1994). It conceived that for healthy follicular development and fertility, both estrogen and androgen are necessary. Casson and colleagues reported that the method of action for DHEA in oral supplementation on the follicular development and the oocyte quality is by increasing the insulin-like growth factor-I serum concentrations.

The impact of DHEA on the ovaries with the patient group that receives Methotrexate never evaluated. The purpose of this study is to determine, primarily the ovarian damage induced by the Methotrexate in this group of patients with a strong desire of fertility and thus evaluating the ovarian reserves and subsequently to determine if DHEA offers any protective properties (preventive and reversing effects) against the detrimental impacts of Methotrexate.

\section{MATERIALS AND METHOD}

This study conducted in the animal testing lab of the Üsküdar University, Istanbul, between the dates of March 25 and April 4 of 2019. The approval for this stud obtained from the Local Ethics Board for Animal Testings of Üsküdar University, İstanbul, Turkey. In this study, 24, 3-6 months old, 200-300gr, Norveginus species Sprague Dawley strain female rats used. The subjects were chosen at random and studied under three groups. Group 1: is the control group (n:8). On the first day of the study, intracardiac blood withdraws from the subjects residing in this group, and the ovaries of the rats were excised the same day with laparotomy and sent to pathology for a histopathological assessment. Group 2: is the DHEA + MTX group $(\mathrm{n}: 8)$, and $1 \mathrm{mg} / \mathrm{kg}$ of methotrexate was applied intramuscularly at the $1 \mathrm{st}, 3 \mathrm{rd}, 5 \mathrm{th}$, and 7 th days of the study. The rats residing in this group received, as a supplementation, $6 \mathrm{mg} / \mathrm{kg} /$ day DHEA (dissolved in $0,1 \mathrm{ml}$ sesame seed oil) subcutaneously for ten days. One of the subjects expired on day 6. Group 3: is the 
MTX group (n:8), and the rats residing in this group received $1 \mathrm{mg} / \mathrm{kg}$ methotrexate intramuscularly at the 1st, 3rd, 5th, and 7th days of the study. On the 11th day, $2 \mathrm{ml}$ of blood withdrawn from Group 2 and Group 3 for the assessment of AMH levels, and on the same day, their ovaries were excised and sent to pathology for a histopathological examination.

The medicines stored at $+4 \mathrm{C}$ in a refrigerator. The rats were caged individually in a controlled environment (room temperature at $21 \mathrm{C}$ and humidity at $60 \%$ ) with $12 \mathrm{~h}$ light/dark cycles, and they fed ad libitum. As anesthetic during the excision of the ovaries with laparotomy, $10 \%$ ketamine hydrochloride at $80 \mathrm{mg} / \mathrm{kg}$ (Ketalar; Eczacıbaşı, Warner-Lambert, Istanbul, Turkey) and $2 \%$ xylazine hydrochloride at $15 \mathrm{mg} / \mathrm{kg}$ (Rompun; Bayer Health Care LCC, Kansas, KS) applied. The ovaries stabilized in a 10\% formalin solution for pathological assessment after the excision. Before the AMH evaluation, the blood samples stored at $-20 \mathrm{C}$. after the completion of the procedure, the animals were decapitated and disposed of using red medical waste bins.

After the administration of the drugs; primordial, primary, secondary, tertiary, atresia and total follicle counts, histopathological damage scores (follicular cell degeneration, vascular congestion, hemorrhage, edema, inflammation, and overall damage) and $\mathrm{AMH}$ values of the control group compared to the similar indicators from the other two groups.

\section{Mode of administration for MTX:}

As advised by the US Bone, Reproductive and Urologic Drugs Advisory Committee (formerly Reproductive Health Drugs Advisory Committee), administered intramuscularly at the $1 \mathrm{st}, 3 \mathrm{rd}$, 5 th and 7 th day (Emthexates ${ }^{\circledR} 50 \mathrm{mg} / 2 \mathrm{ml}$ flacon, Med-lilaç, Istanbul, Turkey).

Mode of preparation and administration for DHEA:

DHEA (CAYMAN®, Dehydroepiandrosterone \%97 1gr, Farmasina, Istanbul, Turkey), prepared by dissolving in sesame seed oil $(0.01 \mathrm{ml}$ dissolved in $95 \%$ ethanol, mixed with $0.09 \mathrm{ml}$ sesame seed oil) (7).

\section{Histopathological assessments:}

All pathology evaluationsents performed by the same specialist at the Pathology Clinic of Medical Sciences University, Kartal Education and Research Hospital, İstanbul, Turkey. Paraffin blocking was performed 24 hours after the oophorectomy, and afterward, 5-millimeter cross-sections obtained. Follicular activity evaluated by taking five random samples from each ovary. The samples were dyed with Hematoxylin + Eosin and then evaluated under a light microscope (Olympus $\mathrm{BH} 2$ Microscope, Olympus Corporation, Tokyo, Japan). These procedures were performed blindly by the same specialist. All follicles in the ovaries (primordial, primary, secondary, tertiary, and atresia) counted, and the ovarian reserves evaluated (Figure 1). Ovarian damage -including follicular cell degeneration, vascular congestion, hemorrhage, edema and inflammation (neutrophil infiltration) -was scored histologically using a graduated scale $(0=$ none, $1=$ mild, $2=$ moderate, and $3=$ severe) (Figure 1) 


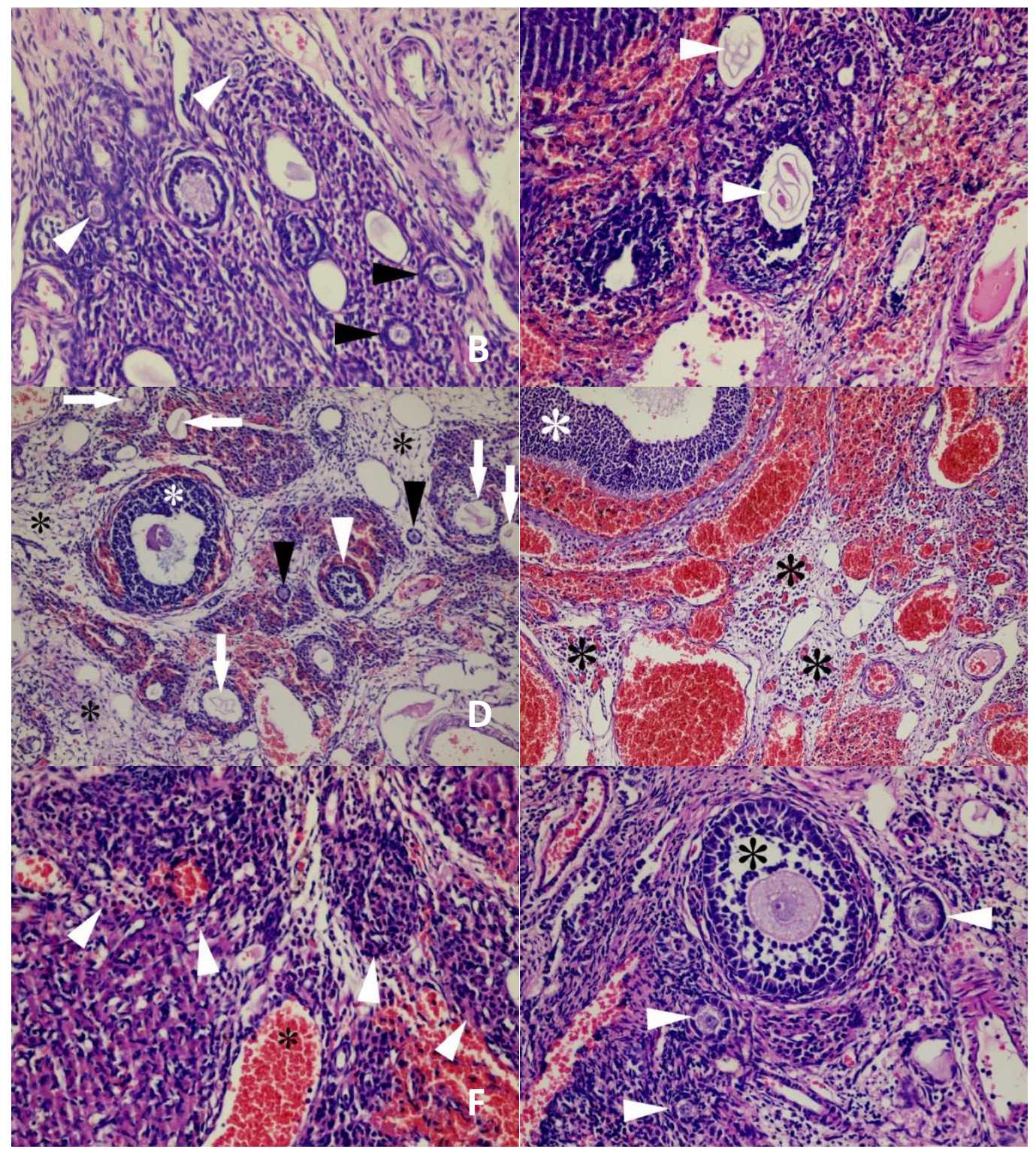

Figure 1: The follicles in the ovaries and the ovarian damage A: Primordial (White arrowheads) and primary follicles (black arrowheads) (H\&E, original magnification x400).B: Degenerate follicles and degenerate oocytes (White arrowheads) (H\&E, original magnification x400). C: Primary (black arrowheads), secondary (White arrowheads), tertiary (White asterisk) follicles and atrophic follicles (White arrows) containing degenerated oocytes (Harrows, Original magnification x200) within a marked edema area (black asterisks).D: Edema (black asterisks), tertiary follicle (White asterisk), multiple congested vessels in between, (H\&E, original magnification x200).E: Inflammatory cells within the ovarian stroma (area surrounded by White arrowheads) and crowded vascular structure (asterisk) (H\&E, original magnification x400).F: Anthral follicle (asterisk) and primary follicles (White arrowheads) (H\&E, original magnification $\mathrm{x} 400)$.

\section{AMH measurement:}

The blood was drawn through injection only at the end of the 1st day in the control group and at the end of the 11th day in the other groups intracardially during the operation after the ovaries excised. The blood samples placed in heparinized tubes (venotect@Italy). Blood was centrifuged in 30 minutes after it draws (15 
minutes $1000 \times \mathrm{g}$ ). The serum removed, and the remaining plasma was placed in an Eppendorf tube and kept frozen at $-20{ }^{\circ} \mathrm{C}$ until the day to be analyzed. AMH levels were measured from plasma in the form of " $\mathrm{ng} / \mathrm{ml}$ " by a biochemist blind to the groups in University of Health Sciences, Dr. Lütfi Kırdar Kartal Training and Research Hospital, Department of Biochemistry, İstanbul/Turkey by using the ELISA (Gemini190605V2, Diatron MI Zrt, Hungary) method. All the samples tested in the same experiment. A kit with a sensitivity of $10 \mathrm{ng} / \mathrm{mL}$, a detection range of $16-10$ $\mathrm{ng} / \mathrm{mL}$, and a variability coefficient of less than $10 \%$ used for the rat $\mathrm{AMH}$ kit (Elabscience, Rat AMH kit; Houston, Texas, ABD).

\section{Statistical analysis:}

For the statistical analysis of the samples, IBM SPSS Statistics version 24 utilized. Shapiro-Wilk and Kolmogorov Smirnov tests used to determine the normality of the samples. While, with the independent groups that conform with normal distribution, one-way variance analysis (ANOVA) utilized for the comparison between three or more groups; for the groups with deviations from the normal distribution, following the Kruskal-Wallis test for three or more comparison groups, the binary comparisons performed with Bonferroni adjusted Mann-Whitney test. After the Bonferroni correction, $p<0.0167$ was accepted to be statistically significant. Between the control group and the others, with the consideration of probable ovarian damage that will be caused by MTX, ROC curve analysis performed to establish an estimate for $\mathrm{AMH}$ level. The patients then divided into two groups based upon the estimate values found. Later, to determine the impact of total ovarian damage and subgroups and total follicle count and subgroups has on the AMH levels, and to establish the appropriate model binary logistic regression analysis performed. Correlation analysis performed to establish whether there was a linear relationship between the dependent variable $\mathrm{AMH}$ and the continuous variables. To determine if variations in the dependent variable were caused by the independent variables, with appropriate models and conditions, the Univariate Covariate Analysis of Variance (ANCOVA) test performed. A p-value of less than 0.05 considered statistically significant.

\section{RESULTS AND DISCUSSION}

The groups studied under three subgroups as the control, the DHEA + MTX, and the MTX groups. The groups compared in regards to their ovarian reserve (OR) (follicle counts and AMH values) and ovarian damage scores (Table 1). In Group 2 and Group 3, the decline of total follicle count and AMH values observed. However, the drop of the primordial, primary, and complete follicle counts and AMH values considered to be statistically significant $(p<0.05)$. In regards to the reduction in the ovarian reserve, when studied in binary comparison; while the considerable decline in the AMH level continued both in the DHEA + MTX and the MTX groups comparative to the control group, it shown that the primordial, primary and total follicle counts have stayed statistically the same in the DHEA + MTX group $(\mathrm{p}<0.0167)$ (Table 2).

The data are presented in average $\pm S D$, or on average \pm Std. Error $\left({ }^{*}\right)$ or in $n$ (\%). For statistics, a: ANOVA, b: Kruskal Wallis Test, c: $x 2$ test, and d: Univariate analysis used. ${ }^{*}$ For corrected $\mathrm{AMH}$ values, the ANCOVA test was used (total damage $=0.78$ and atretic follicle $=0.35$ ). Ovarian damage -including follicular cell degeneration, vascular congestion, hemorrhage, edema and inflammation (neutrophil infiltration) -was scored histologically using a graduated scale $(0=$ none, $1=$ mild, $2=$ moderate, and $3=$ severe) 
Table 1: Intergroup Comparisons of Follicle Counts, Ovarian Damage Scores and $\mathrm{AMH}$ Values

\begin{tabular}{|c|c|c|c|c|c|}
\hline \multicolumn{2}{|c|}{ Parameter/Score } & $\begin{array}{c}\text { Group1 } \\
(n=8)\end{array}$ & $\begin{array}{c}\text { Group2 } \\
(n=7)\end{array}$ & $\begin{array}{c}\text { Group3 } \\
(n=8)\end{array}$ & $\mathrm{p}$ \\
\hline \multicolumn{2}{|c|}{ Primordial follicle } & $5.75 \pm 3.88$ & $1.86 \pm 2.27$ & $1.50 \pm 0.93$ & $.008^{b}$ \\
\hline \multicolumn{2}{|c|}{ Primary follicle } & $11.75 \pm 6.36$ & $6.00 \pm 5.13$ & $4.38 \pm 2.97$ & $.033^{b}$ \\
\hline \multicolumn{2}{|c|}{ Secondary follicle } & $5.50 \pm 2.67$ & $5.00 \pm 4.16$ & $4.75 \pm 2.50$ & $.454^{b}$ \\
\hline \multicolumn{2}{|c|}{ Tertiary follicle } & $9.38 \pm 4.21$ & $6.71 \pm 1.60$ & $4.63 \pm 4.60$ & $.054^{\mathrm{b}}$ \\
\hline \multicolumn{2}{|c|}{ Atretic follicle } & $0.13 \pm 0.35$ & $0.29 \pm 0.49$ & $0.63 \pm 0.52$ & $.112^{b}$ \\
\hline \multicolumn{2}{|c|}{ Total follicle } & $32.38 \pm 13.16$ & $19.57 \pm 10.18$ & $15.25 \pm 11.95$ & $.037^{\mathrm{b}}$ \\
\hline \multirow{2}{*}{ Hemorrhage } & 0 & $8(100)$ & $7(100)$ & $8(100)$ & \multirow{2}{*}{-} \\
\hline & 1 & 0 & 0 & 0 & \\
\hline \multirow{2}{*}{$\begin{array}{c}\text { Vascular } \\
\text { congestion }\end{array}$} & 0 & $3(37.5)$ & $5(71.5)$ & $8(100)$ & \multirow{2}{*}{$.025^{c}$} \\
\hline & 1 & $5(62.5)$ & $2(28.5)$ & 0 & \\
\hline \multirow{2}{*}{$\begin{array}{c}\text { Cellular } \\
\text { degeneration }\end{array}$} & 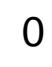 & $7(87.5)$ & $5(71.5)$ & $3(37.5)$ & \multirow{2}{*}{$.101^{\mathrm{c}}$} \\
\hline & 1 & $1(12.5)$ & $2(28.5)$ & $5(62.5)$ & \\
\hline \multirow{2}{*}{$\begin{array}{l}\text { Inflammatory } \\
\text { cell infiltration }\end{array}$} & 0 & $8(100)$ & 7 (100) & $8(100)$ & \multirow{2}{*}{-} \\
\hline & & 0 & 0 & 0 & \\
\hline \multirow{2}{*}{ Edema } & 0 & $5(62.5)$ & $7(100)$ & $8(100)$ & \multirow{2}{*}{$.039^{c}$} \\
\hline & 1 & $3(37.5)$ & 0 & 0 & \\
\hline \multicolumn{2}{|c|}{ Total damage } & $1.13 \pm 0.99$ & $0.57 \pm 0.56$ & $0.63 \pm 0.52$ & $.391^{\mathrm{b}}$ \\
\hline \multicolumn{2}{|c|}{$\mathrm{AMH}(\mathrm{ng} / \mathrm{ml})$} & $2.73 \pm 0.52$ & $0.08 \pm 0.02$ & $0.09 \pm 0.03$ & $.000^{\mathrm{a}}$ \\
\hline \multicolumn{2}{|c|}{$\mathrm{AMH}(\mathrm{ng} / \mathrm{ml})^{*}$} & $2.67 \pm 0.13$ & $0.11 \pm 0.12$ & $0.14 \pm 0.13$ & $.000^{d}$ \\
\hline
\end{tabular}

The data are presented in average \pm SD, or in average \pm Std.Error $\left({ }^{*}\right)$ or in $n(\%)$. For statistics, a: ANOVA, b: Kruskal Wallis Test, c: $x^{2}$ test and $d$ : Univariate test were used. *For corrected AMH values, ANCOVA test was used (total damage $=0.78$ and atretic follicle $=0.35)$. Ovarian damage -including follicular cell degeneration, vascular congestion, hemorrhage, edema and inflammation (neutrophil infiltration) was scored histologically using a graduated scale $(0=$ none, $1=$ mild, $2=$ moderate, and $3=$ severe)

When the relationship between $\mathrm{AMH}$ and the follicles examined, it established that the best relationship formed with the primordial follicles. A significant positive correlation found between the $\mathrm{AMH}$ levels and the primordial follicle counts (correlation coefficient 0.54-0.55) (Figure 2). When the ROC curve performed to establish an estimated value between the control group and the other two in regards to the $\mathrm{AMH}$ and $\mathrm{OR}$ values, the expected point for the insufficient ovarian reserve was established at $1.06233 \mathrm{mg} / \mathrm{ml}$ for $\mathrm{AMH}$. The sensitivity and the specificity of this value is $100 \%$ (area under the curve:1.000; p: .000) (Figure 3). If we were to acknowledge the control group as normal and assume the ovarian reserves decline in the remaining groups, it is an excellent test that exhibits insufficient ovarian reserve. It found that the was TPR (True Positive Rate) $=$ one, and the was FPR $($ False Positive Rate $)=0$. Thus, the test has a success rate of $100 \%$ in distinguishing between the diminished ovarian reserve and the normal ovarian reserve. 
Table 2: Pairwise Intergroup Comparisons of $\mathrm{AMH}$, Primordial, Primary and Total Follicle

\begin{tabular}{|c|c|c|c|}
\hline Groups and $p$ & $1-2$ & $1-3$ & $2-3$ \\
\hline$p^{1}$ & $<.0001$ & $<.0001$ & 0,998 \\
\hline$p^{2}$ & 0,025 & 0,002 & 0,713 \\
\hline$p^{3}$ & 0,105 & 0,008 & 0,639 \\
\hline$p^{4}$ & 0,082 & 0,015 & 0,321 \\
\hline$P^{5}$ & 0 & 0 & 1 \\
\hline
\end{tabular}
${ }^{5}$ Univariate test. $p^{1} \mathrm{AMH}, \mathrm{p}^{2}$ primordial, $p^{3}$ primary, $p^{4}$ total follicle, $p^{5} \mathrm{AMH}$ corrected by using ANOVA.

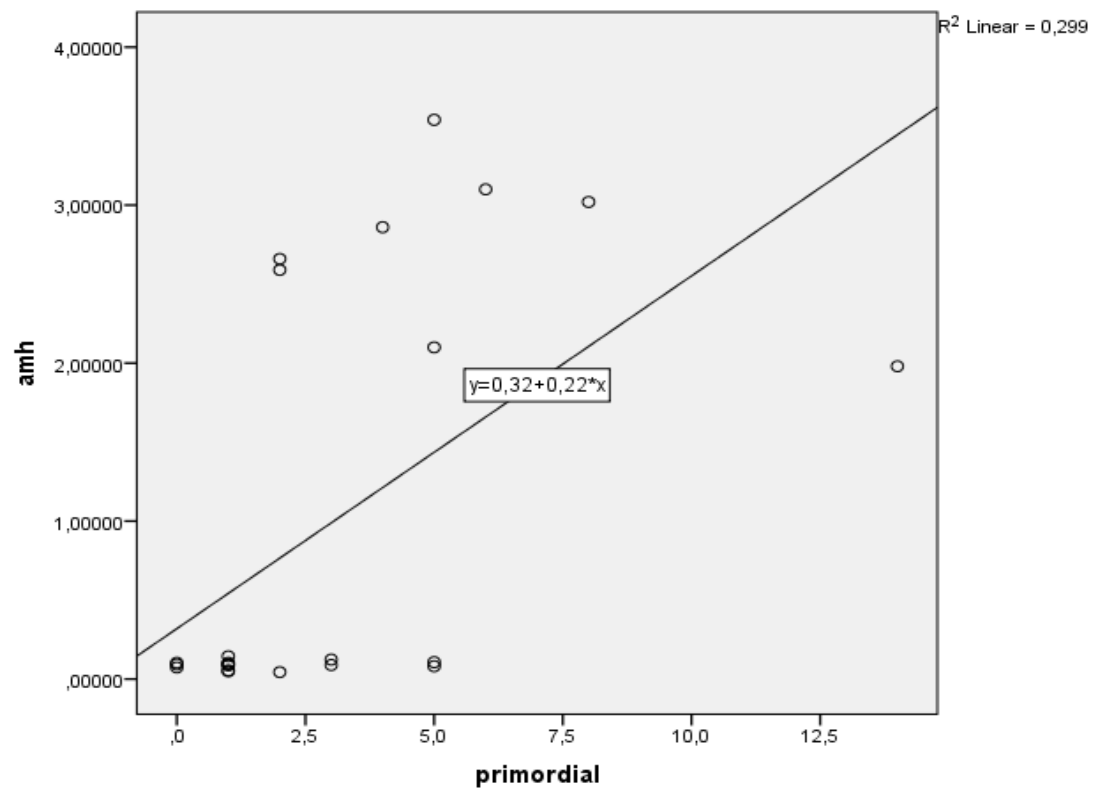

Figure 2: Correlation Between the AMH Levels and The Primordial Follicle Counts

Under this estimation point, a binary group formed, one with diminished ovarian reserve (DOR) and the other with normal ovarian reserve, later, to establish the appropriate independent variables, binary logistic regression analysis performed. When the atresia follicle count and the total damage score models were examined collectively, in regards to their effects on the $\mathrm{AMH}$ level variations between groups, Nagelkerke R-square established at 0.643 , and Cox \& Snell R-square was set at 0.466 and with the $82.6 \%$ rate the conjoint usage of these models found to be acceptable.

Covariance analysis reveals if there are any variations between the $\mathrm{AMH}$ values as the dependent variable amongst the groups. Furthermore, it also shows whether the differences in the dependent variable stems from the differences in the independent variables by subtracting the impact of the independent variables of the total score and the atresia follicle count. When these variables fixed at, the overall rating $=0.78$ and the atresia follicle $=0.35$, it revealed that the distinction, in regards to the $\mathrm{AMH}$ as the dependent variable amongst the groups, stayed the same and it 
revealed to be $p=0,000$ between-group comparison. The variances amongst the groups were between groups 1 and 2 with 1 and 3 .

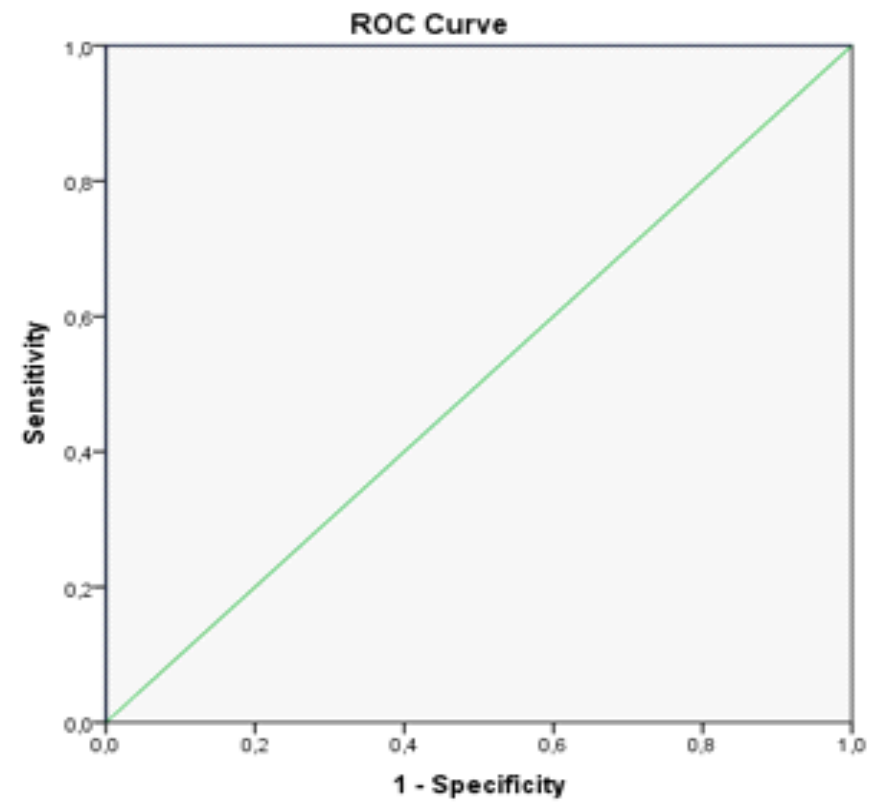

Figure 3: The Sensitivity and The Specificity of The Estimate Point for Insufficient Ovarian Reserve

However, there were no distinctions between groups 2 and 3 , and it established as $p=1,000$. The estimated $\mathrm{AMH}$ value, after the covariance analysis, was found as 2.666 in group 1, 0.110 in group 2, and 0.137 in group 3. The total estimated AMH value found at 0.971 . In conclusion, when the overall damage score and the atresia follicle count fixed, the variance of AMH values between the groups was continuous. Thus DHEA + MTX was found to be insufficient in enhancing the AMH levels and consequently, the ovarian reserve.

Cancers are the primary condition that needs medical intervention and has the potential to impact upon reproductive functions during adolescence. Gonadotoxic treatments, ovarian surgery, premature ovarian insufficiency related to genetic and endocrine disorders are the conditions in which fertility needs to preserve. Evaluation of ovarian reserve is crucial in maintaining fertility in patients. Currently, $\mathrm{AMH}$ is the most commonly used marker while determining ovarian reserves.

$\mathrm{AMH}$ is the marker secreted from granulosa cells of preantral and antral follicles, which can signal the early decline in the ovarian reserves (de Vet, Laven, de Jong, Themmen, \& Fauser, 2002; Tremellen, Kolo, Gilmore, \& Lekamge, 2005). AMH is also a useful marker in determining the effects of chemotherapeutic agents has on the ovarian reserves and in the follow-up after chemotherapy (Oriol et al., 2008; Yeh, Kim, Liang, \& Peresie, 2009). However, the most crucial marker for ovarian reserve is the histological assessment of the ovarian follicles (Oner, Ozcelik, Ozgun, \& Ozturk, 2011). On the other hand, this is not a practical method, and even for research purposes, not always achievable in humans. Hence, the rat model was applied to investigate the effects of the medications on the ovarian reserve (Ozcelik et al., 2010).

Very little known about the impact of MTX on ovarian reserve and the next IVF response of patients, It assumed that MTX is effecting fertility by targeting active 
dividing granulosa cells in ovarian tissue. In return, it means a reduction in ovarian reserve or ovarian response.

The use of adjuvant agents such as DHEA has become prevalent amongst the infertile patients despite the ambiguity about their effectiveness, and this brings controversy. To comprehend the effects DHEA has on ovarian follicles, the developmental process of follicles needs to established. As its known, with the multiplication of granulosa cells, primordial follicles develop into primary follicles, and primary follicles subsequently grow into preantral, antral, and Graafian follicles. The period needed for primordial follicles to develop into Graafian follicles in humans is three months. However, the primordial follicles that represent the ovarian reserves are inactive, and there are no markers that can use to estimate their numbers. Therefore, when speaking of ovarian reserve in clinical practice, instead of primordial follicle pool, it's about the developed antral follicles chosen from this pool (Monniaux et al., 2014).

DHEA considered working by providing recovery from the atresia that effects preantral and small antral follicles that includes improvement in the FSH receptor expression and ovarian IGF-1 factor and additionally by inducing an increase in the antral follicle count (Artini et al., 2012; Casson et al., 1998). However, the effectiveness of DHEA supplementation on DOR is still controversial.

In a study conducted by Bower and colleagues, the relationship between early menopause and the impact of chemotherapy targeting trophoblastic tumors studied, and it reported that usage of methotrexate as a sole agent resulted in menopause two years earlier when compared to patients not receiving chemotherapy (Bower et al., 1998).

In a study performed by Şahin et al., systemic single-dose methotrexate treatment, salpingectomy followed by methotrexate, and salpingectomy alone were applied to ectopic pregnancy cases; AMH levels measured before and after surgery. $\mathrm{AMH}$ levels found to be reassuring, and surgical approaches reportedly did not have any detrimental effects on ovarian reserve (Sahin et al., 2016). Boots and his colleagues also concluded, in a study conducted on ectopic pregnancy cases, that a single dose application of MTX did not have any detrimental effects on the IVF results (Boots, Gustofson, \& Feinberg, 2013). Oriol and colleagues reported that a single dose application of MTX treatment did not endanger ovarian reserve in terms of AMH levels (Oriol et al., 2008). However, in these studies, whether the MTX damages the developing follicles in the ovaries have not been uncovered. Furthermore, these studies conducted on patients who received lower doses of MTX and the effects of multiple MTX does not establish.

Single-dose MTX application is the standard method in the medical treatment of EP. It mostly preferred on unruptured EP cases ("Medical treatment of ectopic pregnancy: A committee opinion," 2013). In the year 2014 Uluğ and Öner (Ulug \& Oner, 2014) have researched the effects of single-dose and multiple-dose MTX applications with salpingectomy on the ovarian reserve; amongst the studied groups, single-dose use of MTX concluded to have the minimal negative impact on the ovarian reserve compared to the other groups; while the primordial follicle counts found comparable between the single-dose MTX group and the control group, they reported that total follicle counts and $\mathrm{AMH}$ levels declined significantly amongst every studied group.

In the study conducted by Göl and colleagues with high doses of MTX on lab rats, it established that high doses of MTX caused damage on the primordial follicles in the ovaries of the rats. The authors reported that this finding could be significant in 
regards to diminished reproductive performance and premature menopause in the following years after high dose MTX applications (Gol, Saygili, Koyuncuoglu, \& Uslu, 2009).

We aimed to determine the probable damage caused by MTX on ovaries and ovarian reserves and also to establish the inhibitory effect of DHEA on these negative aspects as an androgen supplementation. In our study, we saw a significant reduction in the primordial follicle, primary follicle, and total follicle counts at the MTX group comparative to the control group. Once again, in the MTX group, AMH levels were found to be significantly lower compared to the control group. The distinction between our study and the studies done by Şahin, Boots, and Oriol and colleagues is that instead of a single dose application of MTX, in this study, multiple doses of MTX were applied.

Similar to ours, in a study where instead of DHEA, alpha-lipoic acid utilized; it reported that in rats that received MTX, alpha-lipoic acid has reversed the AMH levels and the total follicle counts, and thus healing the oxidative damage to the ovaries. Karapınar and colleagues have concluded that the oxidative effects of MTX on the ovaries may be inhibited by alpha-lipoic acid supplementation which is an antioxidant (Soylu Karapinar, Pinar, Özcan, Özgür, \& Dolapçıoğlu, 2017).

We utilized DHEA instead of alpha-lipoic acid. In the studies conducted until now, DHEA only used in cases with suboptimal ovarian responses or diminished ovarian reserves. However, we applied DHEA supplementation to the rat model with ovarian damage, and diminished ovarian reserves induced my methotrexate. Nonetheless, the DHEA supplementation did not have any significant effects on restoring the AMH levels in the MTX + DHEA group. Additionally, when a binary comparison was made between all the groups, while the $\mathrm{AMH}$ levels were significantly lower in the MTX and the DHEA + MTX groups; primordial follicle, primary follicle, and total follicle counts were reduced considerably only in the MTX group $(\mathrm{p}<0.0167)$.

The reason for DHEA supplementation not providing beneficial effects in our study may be due to the dosage and the timespan it administered. While the follicular recruitment period is three months for humans, it is two months for rats (McGee \& Hsueh, 2000). As the primordial follicles were unaffected in this group, in regards to observing an increase in the AMH levels, extended use of DHEA found to be promising. Studies to this one show that primordial follicles may replace the follicles with developmental deterioration caused by short term MTX treatments for ectopic pregnancies. This phenomenon is explained with the higher resistance of the primordial follicles to ischemia because of their slower metabolic rate, compared to the follicle population in the developmental phase (Liu, Van der Elst, Van den Broecke, Dumortier, \& Dhont, 2000; Nugent, Meirow, Brook, Aubard, \& Gosden, 1997).

Furthermore, we already comprehend that the effects of DHEA are relative to the dosage; as lower doses do not carry any increasing impact on the preantral and antral follicle counts, higher doses may increase the antral follicle counts however it also contributes to the miscarriages due to hyperandrogenism (Mahmoud, Mahmoud, Abo-Zeid, \& Fares, 2018). In rats, DHEA metabolism may differ from humans. Albeit, the dosage of the medication was adjusted to meet with the serum blood level in the human model; however, the inability to measure the DHEA levels in the rat model is the constraint of our study. The lower case count of the groups is another constraint of this study. 


\section{CONCLUSION}

This study demonstrated the toxic effect of MTX, the most widely applied chemotherapeutic agent for malign and benign gynecological disorders. This effect was achieved by multiple doses, while it established that the DHEA treatment was not preventing the ovarian damage caused by MTX, as the primordial follicles seem unaffected in the DHEA group, it offers hope in increasing AMH levels with prolonged usage. As the mechanism of action of DHEA based on folliculogenesis is substantially complicated. The need for randomized prospective studies designed with more full sample groups, various dosages, and different treatment periods is apparent to assess the protective effect of DHEA has on ovarian reserves.

\section{CONFLICT OF INTEREST}

There were no conflicts of interest with related parties in this study.

\section{REFERENCES}

Artini, P. G., Simi, G., Ruggiero, M., Pinelli, S., Di Berardino, O. M., Papini, F., ... Cela, V. (2012). DHEA supplementation improves follicular microenviroment in poor responder patients. Gynecological Endocrinology, 28(9), 669-673. https://doi.org/10.3109/09513590.2012.705386

Boots, C. E., Gustofson, R. L., \& Feinberg, E. C. (2013). Does methotrexate administration for ectopic pregnancy after in vitro fertilization impact ovarian reserve or ovarian responsiveness? Fertility and Sterility, 100(6), 1590-1593. https://doi.org/10.1016/j.fertnstert.2013.08.007

Bower, M., Rustin, G. J., Newlands, E. S., Holden, L., Short, D., Foskett, M., \& Bagshawe, K. D. (1998). Chemotherapy for gestational trophoblastic tumours hastens menopause by 3 years. European Journal of Cancer (Oxford, England: 1990), 34(8), 1204-1207. https://doi.org/10.1016/s0959-8049(98)00059-8

Burger, H. G. (2002). Androgen production in women. Fertility and Sterility, 77(SUPPL. 4), 3-5. https://doi.org/10.1201/b14632-2

Casson, P. R. (2000). Dehydroepiandrosterone supplementation augments ovarian stimulation in poor responders: a case series. Human Reproduction, 15(10), 2129-2132. https://doi.org/10.1093/humrep/15.10.2129

Casson, P. R., Santoro, N., Elkind-Hirsch, K., Carson, S. A., Hornsby, P. J., Abraham, G., \& Buster, J. E. (1998). Postmenopausal dehydroepiandrosterone administration increases free insulin-like growth factor-I and decreases highdensity lipoprotein: a six-month trial. Fertility and Sterility, 70(1), 107-110. https://doi.org/10.1016/s0015-0282(98)00121-6

de Vet, A., Laven, J. S. E., de Jong, F. H., Themmen, A. P. N., \& Fauser, B. C. J. M. (2002). Antimüllerian hormone serum levels: a putative marker for ovarian aging. Fertility and Sterility, 77(2), 357-362. https://doi.org/10.1016/s00150282(01)02993-4

Gol, M., Saygili, U., Koyuncuoglu, M., \& Uslu, T. (2009). Influence of high-dose methotrexate therapy on the primordial follicles of the mouse ovary. Journal of Obstetrics and Gynaecology Research, 35(3), 429-433. https://doi.org/10.1111/j.1447-0756.2008.00945.x

Howell, S., \& Shalet, S. (1998). Gonadal damage from chemotherapy and radiotherapy. Endocrinology and Metabolism Clinics of North America, 27(4), 927-943. https://doi.org/10.1016/S0889-8529(05)70048-7

Lipscomb, G. H. (2007, March). Medical therapy for ectopic pregnancy. Seminars in Reproductive Medicine, Vol. 25, pp. 93-98. https://doi.org/10.1055/s-2007- 


\section{8}

Liu, J., Van der Elst, J., Van den Broecke, R., Dumortier, F., \& Dhont, M. (2000). Maturation of Mouse Primordial Follicles by Combination of Graftingand In Vitro Culture1. Biology of Reproduction, 62(5), 1218-1223. https://doi.org/10.1095/biolreprod62.5.1218

Luchetti, C. G., Solano, M. E., Sander, V., Arcos, M. L. B., Gonzalez, C., Di Girolamo, G., ... Motta, A. B. (2004). Effects of dehydroepiandrosterone on ovarian cystogenesis and immune function. Journal of Reproductive Immunology, 64(1-2), 59-74. https://doi.org/10.1016/j.jri.2004.04.002

Mahmoud, Y. I., Mahmoud, A. A., Abo-Zeid, F. S., \& Fares, N. H. (2018). Effects of dehydroepiandrosterone on the ovarian reserve and pregnancy outcomes in perimenopausal rats (DHEA and fertility in perimenopausal rats). Life Sciences, 199, 131-138. https://doi.org/10.1016/j.lfs.2018.03.021

McGee, E. A., \& Hsueh, A. J. W. (2000). Initial and Cyclic Recruitment of Ovarian Follicles 1 . Endocrine Reviews, 21(2), 200-214. https://doi.org/10.1210/edrv.21.2.0394

Medical treatment of ectopic pregnancy: A committee opinion. (2013). Fertility and Sterility, 100(3), 638-644. https://doi.org/10.1016/j.fertnstert.2013.06.013

Millier, S. G., Whitelaw, P. F., \& Smyth, C. D. (1994). Follicular oestrogen synthesis: the "two-cell, two-gonadotrophin" model revisited. Molecular and Cellular Endocrinology, 100(1-2), 51-54. https://doi.org/10.1016/0303-7207(94)90278-X

Monniaux, D., Clément, F., Dalbiès-Tran, R., Estienne, A., Fabre, S., Mansanet, C., \& Monget, P. (2014). The Ovarian Reserve of Primordial Follicles and the Dynamic Reserve of Antral Growing Follicles: What Is the Link?1. Biology of Reproduction, 90(4). https://doi.org/10.1095/biolreprod.113.117077

Nugent, D., Meirow, D., Brook, P. F., Aubard, Y., \& Gosden, R. G. (1997, May). Transplantation in reproductive medicine: Previous experience, present knowledge and future prospects. Human Reproduction Update, Vol. 3, pp. 267280. https://doi.org/10.1093/humupd/3.3.267

Oner, G., Ozcelik, B., Ozgun, M. T., \& Ozturk, F. (2011). The effects of metformin and letrozole on endometrium and ovary in a rat model. Gynecological Endocrinology, 27(12), 1084-1086. https://doi.org/10.3109/09513590.2011.589928

Oriol, B., Barrio, A., Pacheco, A., Serna, J., Zuzuarregui, J. L., \& Garcia-Velasco, J. A. (2008). Systemic methotrexate to treat ectopic pregnancy does not affect ovarian reserve. Fertility and Sterility, 90(5), 1579-1582. https://doi.org/10.1016/j.fertnstert.2007.08.032

Ozcelik, B., Turkyilmaz, C., Ozgun, M. T., Serin, I. S., Batukan, C., Ozdamar, S., \& Ozturk, A. (2010). Prevention of paclitaxel and cisplatin induced ovarian damage in rats by a gonadotropin-releasing hormone agonist. Fertility and Sterility, 93(5), 1609-1614. https://doi.org/10.1016/j.fertnstert.2009.02.054

Sahin, C., Taylan, E., Akdemir, A., Ozgurel, B., Taskıran, D., \& Ergenoglu, A. M. (2016). The impact of salpingectomy and single-dose systemic methotrexate treatments on ovarian reserve in ectopic pregnancy. European Journal of Obstetrics and Gynecology and Reproductive Biology, 205, 150-152. https://doi.org/10.1016/j.ejogrb.2016.08.028

Soylu Karapinar, O., Pinar, N., Özcan, O., Özgür, T., \& Dolapçığlu, K. (2017). Protective effect of alpha-lipoic acid in methotrexate-induced ovarian oxidative injury and decreased ovarian reserve in rats. Gynecological Endocrinology, 33(8), 653-659. https://doi.org/10.1080/09513590.2017.1306847 
Tremellen, K. P., Kolo, M., Gilmore, A., \& Lekamge, D. N. (2005). Anti-müllerian hormone as a marker of ovarian reserve. Australian and New Zealand Journal of Obstetrics and Gynaecology, 45(1), 20-24. https://doi.org/10.1111/j.1479828X.2005.00332.x

Ulug, P., \& Oner, G. (2014). Evaluation of the effects of single or multiple dose methotrexate administration, salpingectomy on ovarian reserve of rat with the measurement of anti-Müllerian hormone $(\mathrm{AMH})$ levels and histological analysis. European Journal of Obstetrics and Gynecology and Reproductive Biology, 181, 205-209. https://doi.org/10.1016/j.ejogrb.2014.07.011

Wallace, W. H. B. (2011). Oncofertility and preservation of reproductive capacity in children and young adults. Cancer, 117(SUPPL. 10), 2301-2310. https://doi.org/10.1002/cncr.26045

Yeh, J., Kim, B. S., Liang, Y. J., \& Peresie, J. (2009). Gonadotropin stimulation as a challenge to calibrate cisplatin induced ovarian damage in the female rat. Reproductive Toxicology, 28(4), $556-562$. https://doi.org/10.1016/j.reprotox.2009.08.003 